\title{
Evaluation of the Unperturbed Dimension by Using Potential Energy Surface
}

\author{
Tomo-o OYAMA and Kohzoh SHIOKAWA \\ Department of Applied Science, Faculty of Engineering, \\ Kyushu University, Hakozaki, Higashi-ku, \\ Fukuoka 812, Japan.
}

(Received June 24, 1981)

\begin{abstract}
KEY WORDS End-to-End Distance / Averages of Bond Conformation / Rotational Isomeric State Approximation / Integral Equation /
\end{abstract}

One of the important quantities characterizing a linear polymer chain is the mean-square end-to-end distance $\left\langle R^{2}\right\rangle$. In the unperturbed state, $\left\langle R^{2}\right\rangle$ is closely related to the internal rotation around skeletal bonds. It is well known in regard to the theoretical estimation of $\left\langle R^{2}\right\rangle$ that not only the interaction between the nearest neighbouring groups along the chain, but also steric hindrance caused by the second neighbours have important effect. The latter effect, called the correlated interaction or the $\mathrm{GG}^{*}$ effect, was taken into account by $\mathrm{Nagai}^{1}$ and by Lifson, ${ }^{2}$ and was reviewed by Flory. ${ }^{3}$ In their treatments, the rotational isomeric state approximation (RISA) was adopted. The validity of this approximation was examined for the case in which the correlated interaction can be ignored. ${ }^{4}$ However, there are only a few studies on the validity of this approximation for the case where the correlated interaction is present.

The method for calculating the averages of the transformation matrix product with no recourse to RISA has been formulated by Saitô. ${ }^{5}$ In his method, averages are calculated by using eigenvalues and eigenfunctions of the integral equation whose kernel corresponds to the statistical weight of a rotational state. But the integral equation cannot be solved analytically unless the correlated interaction is negligible.

In this short note, the integral equation is solved numerically for the model potential field and $\left\langle R^{2}\right\rangle$ 's are evaluated. A discussion on the validity of RISA is made.

\section{THEORETICAL}

Skeletal bond vectors of a polymer chain are denoted by $\boldsymbol{b}_{1}, \boldsymbol{b}_{2}, \cdots$. All bond-lengths and bondangles are held fixed to the same constants, denoted by $b$ and $\theta$, respectively. As is well known, the mean-square end-to-end distance is given by,

$$
\left\langle R^{2}\right\rangle=n b^{2}+2 \sum_{j>i}\left\langle\boldsymbol{b}_{i} \cdot \boldsymbol{b}_{j}\right\rangle
$$

where $n$ is the total number of bonds, and the averages are taken over all the possible conformations of the chain. Using the three-dimensional orthogonal matrix $\boldsymbol{T}_{i}$ which transforms the vector $\boldsymbol{b}_{i}$ to the vector $\boldsymbol{b}_{i+1}$ in eq 1, we obtain for the characteristic ratio $C_{\infty}$,

$$
C_{\infty} \equiv\left\langle R^{2}\right\rangle / n b^{2}=1+2 \tilde{\boldsymbol{e}} \sum_{j>i} \sum_{j-1}\left\langle\boldsymbol{T}_{j-1} \cdots \boldsymbol{T}_{i}\right\rangle \boldsymbol{e} / n
$$

where $\boldsymbol{e}$ is an unit vector and $\tilde{\boldsymbol{e}}$ is the its transpose. The average of the transformation matrix product is expressed by

$$
\begin{aligned}
& \left\langle\boldsymbol{T}_{j-1} \cdots \boldsymbol{T}_{i}\right\rangle= \\
& \frac{\int \cdots \int \boldsymbol{T}_{j-1} \cdots \boldsymbol{T}_{i} \exp (-\beta U) \mathrm{d} \phi_{1} \mathrm{~d} \phi_{2} \cdots \mathrm{d} \phi_{n}}{\int \cdots \int \exp (-\beta U) \mathrm{d} \phi_{1} \mathrm{~d} \phi_{2} \cdots \mathrm{d} \phi_{n}}
\end{aligned}
$$

where $U$ is the total conformation energy. The integration extends over all the rotational angles $\phi_{i}$. We assume that $U$ is expressed as, 


$$
U=\sum_{i} U_{1}\left(\phi_{i}\right)+\sum_{i} U_{2}\left(\phi_{i}, \phi_{i+1}\right)
$$

where $U_{1}\left(\phi_{i}\right)$ is the interaction energy between the nearest neighbours and $U_{2}\left(\phi_{i}, \phi_{i+1}\right)$ is the hindrance energy caused by the second neighbours. Higher-order terms are neglected.

According to Saitô, ${ }^{5}$ we introduce an integral equation,

$$
\lambda_{m} \psi_{m}\left(\phi_{1}\right)=\int K\left(\phi_{1}, \phi_{2}\right) \psi_{m}\left(\phi_{2}\right) \mathrm{d} \phi_{2}
$$

where $\lambda_{m}$ is the $m$-th eigenvalue, and $\psi_{m}(\phi)$ is its eigenfunction. The kernel $K$ is defined by,

$K\left(\phi_{1}, \phi_{2}\right)=$

$$
\exp \beta\left\{-\frac{1}{2} U_{1}\left(\phi_{1}\right)-\frac{1}{2} U_{1}\left(\phi_{2}\right)-U_{2}\left(\phi_{1}, \phi_{2}\right)\right\}
$$

where $\beta=1 / k T$. Since the kernel $K\left(\phi_{1}, \phi_{2}\right)$ is symmetric with respect to $\phi_{1}$ and $\phi_{2}$, it can be expanded as

$$
K\left(\phi_{1}, \phi_{2}\right)=\sum_{m} \lambda_{m} \psi_{m}\left(\phi_{1}\right) \psi_{m}\left(\phi_{2}\right)
$$

By using the orthonormality of $\psi_{m}(\phi)$ and the cyclic condition, $\phi_{n+1}=\phi_{1}$, we can rewrite eq 3 as,

$$
\left\langle\boldsymbol{T}_{j-1} \cdots \boldsymbol{T}_{i}\right\rangle=\frac{\sum_{m} \lambda_{m}{ }^{n-(j-i)}\left|\boldsymbol{F}^{j-i}\right|_{m m}}{\sum_{m} \lambda_{m}{ }^{n}}
$$

where $\boldsymbol{F}$ is a square matrix whose element is defined as

$$
\boldsymbol{F}_{i j}=\lambda_{j} \int \psi_{i}(\phi) \boldsymbol{T}(\phi) \psi_{j}(\phi) \mathrm{d} \phi
$$

and ||$_{m m}$ denotes the $m, m$ element of the matrix. If we let $n$ approach infinity, all the terms related to $\lambda_{m}{ }^{n}$ become negligible, except for the terms related to the largest eigenvalue, $\lambda_{M}{ }^{n}$. Thus from eq 2, 6 and 7 we have,

$$
C_{\infty}=2 \tilde{\boldsymbol{e}}\left(\boldsymbol{E}-\frac{\boldsymbol{F}}{\lambda_{M}}\right)_{M M}^{-1} \boldsymbol{e}-1
$$

where $\boldsymbol{E}$ is an unit matrix.

To calculate the average values of functions of the internal rotation angles, we must solve eq 5 . Though the equation cannot be solved analytically, its numerical solution can be obtained as follows. The integral of a function $f(x)$ is evaluated as a weighted sum of the values of the function at sampling points, i.e.,

$$
\int f(x) \mathrm{d} x=\sum_{j} H_{j} f\left(x_{j}\right),
$$

where $H_{j}$ is the weight at abscissa $x_{j}$. The values of $H_{j}$ and $x_{j}$ depend on the quadrature formula adopted.* Hence, eq 5 can be rewritten as,

$$
\lambda_{m} \psi_{m}\left(\phi_{i}\right)=\int K\left(\phi_{i}, \phi\right) \psi_{m}(\phi) \mathrm{d} \phi=\sum_{j} H_{j} K\left(\phi_{i}, \phi_{j}\right) \psi_{m}\left(\phi_{j}\right)
$$

By multipling both sides of this equation by $H_{i}{ }^{1 / 2}$, the following equation is obtained:

$$
\begin{aligned}
\lambda_{m}\left[H_{i}{ }^{1 / 2} \psi_{m}\left(\phi_{i}\right)\right] & \\
& =\sum_{j}\left[H_{i}{ }^{1 / 2} H_{j}{ }^{1 / 2} K\left(\phi_{i}, \phi_{j}\right)\right]\left[H_{j}{ }^{1 / 2} \psi_{m}\left(\phi_{j}\right)\right]
\end{aligned}
$$

Equation 9 is expressed in matrix representation as,

$$
\lambda_{m} \boldsymbol{u}_{m}=\boldsymbol{G} \boldsymbol{u}_{m}
$$

The $i$-th component of $\boldsymbol{u}_{m}$ is $H_{i}{ }^{1 / 2} \psi_{m}\left(\phi_{i}\right)$ and the $i, j$ element of $\boldsymbol{G}$ is $H_{i}^{1 / 2} H_{j}^{1 / 2} K\left(\phi_{i}, \phi_{j}\right)$. If the values of $U_{1}(\phi)$ and $U_{2}\left(\phi_{i}, \phi_{j}\right)$ are estimated at rotational angles $\phi_{i}(i=1,2, \cdots n)$, all of the elements of $\boldsymbol{G}$ can be calculated. Since $\boldsymbol{G}$ is a real symmetric matrix, eq 9 , which is the familiar eigenvalue problem, can be solved numerically stable by use of standard procedures. The component of the mean value of function $f(\phi)$ can be evaluated as,

$$
\begin{aligned}
& \langle f(\phi)\rangle_{m n}= \\
& \int \psi_{m}(\phi) f(\phi) \psi_{n}(\phi) \mathrm{d} \phi \simeq \sum_{j} H_{j} \psi_{m}\left(\phi_{j}\right) f\left(\phi_{j}\right) \psi_{n}\left(\phi_{j}\right) \\
& \quad=\sum_{j} \boldsymbol{u}_{m}{ }^{(j)} f\left(\phi_{j}\right) \boldsymbol{u}_{n}^{(j)}
\end{aligned}
$$

In the same manner, all elements of $\boldsymbol{F}$ in eq 8 can be evaluated by eq 7 , and $C_{\infty}$ can be calculated by eq 8 .

\section{RESULTS AND DISCUSSION}

Polymethylene was adopted as a model chain. The conformational energy of this chain was calcu-

* RISA corresponds to the case, $\phi_{1}=\phi_{\mathrm{G}}, \phi_{2}=\phi_{\mathrm{T}}$, $\phi_{3}=\phi_{\mathrm{G}^{*}}$, and $H_{1}=H_{2}=H_{3}=1 / 3$. 


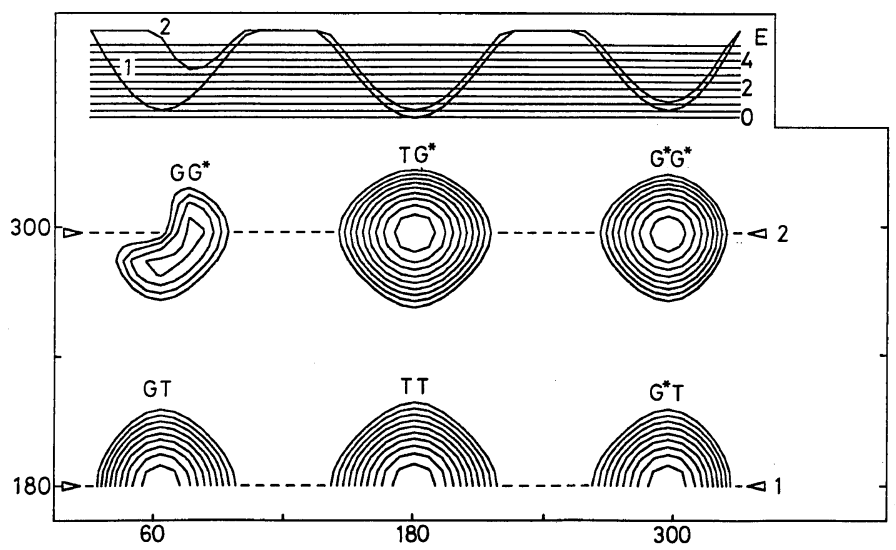

Figure 1. An example of the conformational energy map of the model chain. Contours are shown at interval of $0.5 \mathrm{kcal} \mathrm{mol}^{-1}$. The minimum energy of each region relative to the TT region is adjusted to $E_{\mathrm{TG}}=0.55, E_{\mathrm{GG}}=1.10, E_{\mathrm{GG}^{*}}=3.30 \mathrm{kcal} \mathrm{mol}^{-1}$, respectively. Cross sections, shown at upside, correspond to the rotational angles indicated with dashed lines.

lated by using the threefold rotational barriers and the 6-exp Buckingham type non-bonded interactions. The values used for the parameters of the potential functions and for the molecular parameters were the same as those given by Abe, Jernigan, and Flory. ${ }^{6}$ To adjust the energy differences between the minima, the non-bonded interactions were multiplied by suitable chosen scaling parameters. An example of numerical computation is as follows. The quadrature used was 47 points Simpson's quadrature, and the range of integration was from $31^{\circ}$ to $329^{\circ}$. Eigenvalues and eigenvectors in eq 10 were calculated using QR algorithm. ${ }^{7}$ All computations were carried on the M-200 of Computer Center, Kyushu University.

Figure 1 shows an example of the conformational energy contour map. In this map, contours are drawn at intervals of $0.5 \mathrm{kcal} \mathrm{mol}^{-1}$, and those above $3 \mathrm{kcal} \mathrm{mol}^{-1}$ are omitted. The value of the minimum energy in each region, relative to that of the TT region, is adjusted to $E_{\mathrm{TG}}=0.55, E_{\mathrm{GG}}=1.10$, and $E_{\mathrm{GG}^{*}}=3.30$ in $\mathrm{kcal} \mathrm{mol}^{-1}$. These minima are located at $180^{\circ}$ for $T$ and $\pm 63.5^{\circ}$ for $G$ in the TT, TG, and GG regions, and a pair of the equivalent minima is located at $\left(78.8^{\circ},-63.0^{\circ}\right)$ and $\left(63.0^{\circ}\right.$, $\left.-78.8^{\circ}\right)$ in the GG* region, as has been shown by Abe et al. ${ }^{6}$ The value of the mean energy at $140^{\circ} \mathrm{C}$ in each region, relative to that of the TT region, is $\bar{E}_{\mathrm{TG}}=0.54, \bar{E}_{\mathrm{GG}}=1.08$, and $\bar{E}_{\mathrm{GG}^{*}}=3.11$ in $\mathrm{kcal} \mathrm{mol}^{-1}$. The mean values of the rotational angles are $180^{\circ}$ for $T$ and $64.2^{\circ}$ for $G$ in the TT, TG, and $\mathrm{GG}$ regions, and are $\pm 75.5^{\circ}$ in the $\mathrm{GG}^{*}$ region. It is noteworthy that the differences between the minima and the mean values for the energies and the rotational angles are very small except in the $\mathrm{GG}^{*}$ region which has a pair of minima.

The probability density $\rho(\phi)$ at rotational angle $\phi_{i}$ is defined as,

$$
\rho(\phi)=\frac{\int \exp (-\beta U) \mathrm{d} \phi_{1} \cdots \mathrm{d} \phi_{i-1} \mathrm{~d} \phi_{i+1} \cdots \mathrm{d} \phi_{n}}{\int \exp (-\beta U) \mathrm{d} \phi_{1} \cdots \mathrm{d} \phi_{n}}
$$

Exp $(-\beta U)$ can be expanded using $\lambda_{m}$ and $\psi_{m}(\phi)$. With this use the integration can be done easily. We obtain for $\rho(\phi)$,

$$
\rho(\phi)=\sum_{m} \lambda_{m}{ }^{n} \psi_{m}{ }^{2}(\phi) / \sum_{m} \lambda_{m}{ }^{n} \simeq \psi_{M}(\phi)
$$

The probability density and the statistical weight $\mathrm{e}^{-\beta U_{1}(\phi)}$ at $140^{\circ} \mathrm{C}$ are shown as functions of $\phi$ in Figure 2 . The probability density is lower than the statistical weight in the $\mathrm{G}$ region for a long chain, as has been pointed by Flory who used RISA. ${ }^{3}$

In Table I, the average bond conformations are given, where $f_{\mathrm{I}}$ is the probability of a bond found in the $\mathrm{I}$ region and $f_{\mathrm{IJ}}$ is the probability of two adjacent bonds found in the IJ region. $f_{\mathrm{T}}$ is about $15 \%$ smaller than that expected by RISA. But $f_{\mathrm{T}}$ is larger than the statistical weight of the $T$ region, as shown in Figure 2. The value of $f_{\mathrm{G}}$ is larger than that 


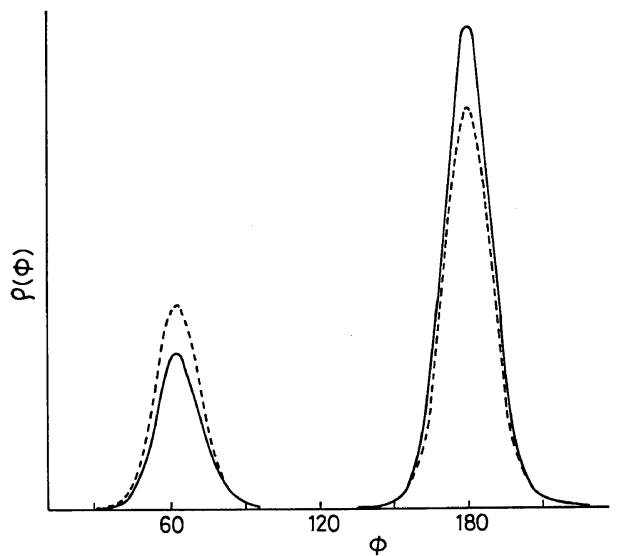

Figure 2. Probability density at $140^{\circ} \mathrm{C}$ as a function of rotational angle: solid line, probability density; dashed line, statistical weight.

Table I. Averages of bond conformations of the model chain at $140^{\circ} \mathrm{C}$

\begin{tabular}{lrrr} 
& & \multicolumn{2}{c}{ Rot. isomer model } \\
\cline { 3 - 4 } & Present & \multicolumn{2}{c}{ method } \\
\cline { 3 - 4 } & & \multicolumn{1}{c}{$\min$} & mean \\
\hline$f_{\mathrm{T}}$ & 0.618 & 0.723 & 0.718 \\
$f_{\mathrm{G}}$ & 0.382 & 0.277 & 0.282 \\
$\langle\cos \phi\rangle$ & -0.488 & -0.599 & -0.592 \\
$f_{\mathrm{TT}}$ & 0.351 & 0.499 & 0.493 \\
$f_{\mathrm{TG}}$ & 0.534 & 0.477 & 0.451 \\
$f_{\mathrm{GG}}$ & 0.101 & 0.050 & 0.052 \\
$f_{\mathrm{GG}}$ & 0.012 & 0.003 & 0.004 \\
\hline
\end{tabular}

expected by RISA and smaller than the statistical weight of the $G$ region. The mean value of $\cos \phi$, $\langle\cos \phi\rangle$, by the present calculation is different from that by RISA. This is partially due to the difference $f_{\mathrm{T}}$ (and also in $f_{\mathrm{G}}$ ) stated above, but another reason is that $\langle\cos \phi\rangle$ is very different from $\cos \langle\phi\rangle$ in the $T$ region. The fractions of the bond pairs are shown in the fourth to the seventh column of Table I. The value of $f_{\mathrm{TT}}$ by the present calculation is quite different from that by RISA. The values of $f_{\mathrm{GG}}$ and $f_{\mathrm{GG}^{*}}$ in the present calculation are two to four times larger than those of RISA. RISA is not a good approximation for the averages of bond conformations. This means that the averages of bond conformations are sensitive to the shape of potential surface near minima.

The calculated value of $C_{\infty}$ and its temperature
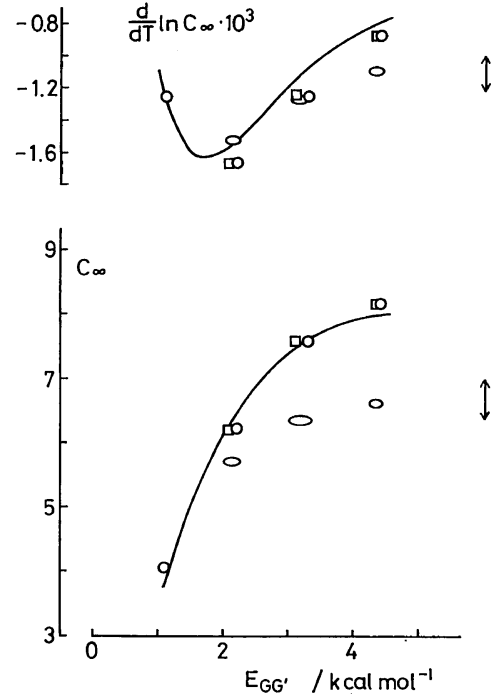

Figure 3. Characteristic ratio $C_{\infty}$ and its temperature dependence at $140^{\circ} \mathrm{C}$ as functions of $E_{\mathrm{GG}^{*}}$; calculated values are plotted against to minima energies $\left(E_{\mathrm{GG}^{*}}, \bigcirc\right)$ and to average energies $\left(\bar{E}_{\mathrm{GG}^{*}}, \square\right)$, respectively; ellipses are calculated by the perturbation calculation; solid curves are calculated on RISA $\left(\phi_{\mathrm{G}}=63.5^{\circ}\right)$.

dependence, $(\mathrm{d} / \mathrm{d} T) \ln C_{\infty}$ are plotted against $E_{\mathrm{GG}^{*}}$ in Figure 3 for the case where $E_{\mathrm{TG}}=0.55 \mathrm{kcal} \mathrm{mol}^{-1}$ and $E_{\mathrm{GG}}=1.10 \mathrm{kcal} \mathrm{mol}^{-1}$ at $140^{\circ} \mathrm{C}$. The points indicated by circles and squares in the figure represent the values obtained by using the minima and the mean energy of the GG* region, respectively. The curves are calculated using RISA corresponding $\phi_{\mathrm{G}}=63.5^{\circ}$. Arrows in the right-hand-side of the figures indicate the experimental values cited by Flory. The agreement between $C_{\infty}$ by the present method and RISA is very good, though the values of $C_{\infty}$ are very sensitive to $E_{\mathrm{GG}^{*}}$ and $\phi_{\mathrm{G}}$. For (d/d $T) \ln C_{\infty}$, the calculated values by RISA are about $10 \%$ larger than that by the present method. But, taking into account the fact that the values of (d/d $T) \ln C_{\infty}$ is sensitive to $E_{\mathrm{GG}^{*}}$ as shown in Figure 3. We may conclude that the results by the two methods are in fair agreement. In practice, RISA is a very simple and useful approximation for calculating $\left\langle R^{2}\right\rangle$. This may be due to the fact that $\left\langle R^{2}\right\rangle$ is sensitive to the locations and relative stabilities of potential minima, but insensitive to the shape of potential energy surface.

The ellipses in Figure 3 indicate the calculated values using the perturbation method developed by 
Takahashi, Kotera, and Miyake. ${ }^{8}$ The discrepancy between the present method and the perturbation method became appreciable as $E_{\mathrm{GG}^{*}}$ increases. This is because the basic assumption of the perturbation method, $\exp \left(-\beta U_{2}\left(\phi_{1}, \phi_{2}\right)\right)-1 \ll 1$, is not appropriate when the value of $E_{\mathrm{GG}^{*}}$ is large.

\section{CONCLUSION}

The end-to-end distance and averages of bond conformations were calculated without using the rotational isomeric state approximation. An integral equation, whose kernel corresponds to statistical weights of rotational angles, was solved numerically for a model polymethylene chain.

We conclude that the rotational isomeric state approximation is very simple and useful for the calculation of $\left\langle R^{2}\right\rangle$, but is not suitable for calculating the averages of bond conformations.

\section{REFERENCES}

1. K. Nagai, J. Chem. Phys., 31, 1169 (1959).

2. S. Lifson, J. Chem. Phys., 30, 964 (1959).

3. P. J. Flory, "Statistical Mechanics of Chain Molecules," Interscience, New York, N.Y., 1969.

4. M. V. Volkenstein, "Configurational Statistics of Polymeric Chains," English Translation, S. N. Timasheff and M. J. Timasheff, Interscience, New York, N.Y., 1969.

5. N. Saitô, "Kohbunshi no Butsuri," Physical Society of Japan, Ed., Asakura, Tokyo, 1963 (in Japanese); N. Saitô, K. Okano, S. Iwayanagi, and T. Hideshima, Solid State Phys., 14, 344 (1963).

6. A. Abe, R. L. Jernigan, and P. J. Flory, J. Am. Chem. Soc., 88, 631 (1966).

7. for example; H. Bowdler, R. S. Martin, C. Reinsch, and J. H. Wilkinson, Numer. Math., 11, 293 (1968).

8. N. Takahashi, A. Kotera, and A. Miyake, Rep. Prog. Polym. Phys. Jpn., 17, 21 (1974). 